\title{
Automatic Car License Plate Detection System for Odd and Even Series
}

\author{
Sapna Gaur \\ Research Scholar \\ Hindustan Institute of \\ Technology Agra APJ Abdul \\ Kalam Technical University, Lucknow
}

\author{
Sweta Singh \\ Asst. Professor \\ Hindustan Institute of \\ Technology AgraAPJ Abdul \\ Kalam Technical University, Lucknow
}

\begin{abstract}
An automatic car license plate detection system is essential for today's busy traffic as it allows for quick traffic monitoring, toll processing and law enforcements related to traffic. Over the years many researchers have successfully developed much automatic car license plate detection system. Each system has its own set of advantages and limitations. In India, the need for automatic car license plate detection system is highly essential. Currently States like Delhi have adopted an Odd-even based traffic policy to bring down the levels of traffic pollution. In such scenarios it becomes highly difficult for the traffic inspectors to manually monitor oddeven series car plates. Thus, this work is a preliminary effort in developing an automatic car license plate detection system for odd-even based systems and it also supports the classification of car license based on the color of the license plate like private vehicle, commercial vehicle, government vehicle and so on. This work makes use of integration of SURF, Multiclass SVM and OCR for car license plate detection system. The proposed system is very fast and accurate results are obtained in less time. The system on execution successfully classifies the odd and even vehicles with an accuracy of $94 \%$.
\end{abstract}

\section{Keywords}

Car License plate detection, Odd even system, SVM, SURF, OCR

\section{INTRODUCTION}

Automatic license detection system is an essential system in today's busy traffic system. It helps in automatic monitoring of traffic rules and other law enforcement activities. In India there are many cases of rash driving where in cars violating many traffic rules. It becomes very difficult for traffic policeman to capture the details of the vehicle. Thus, automatic license detection system have been proposed and implemented over the years to help easy and fast monitoring of traffic rules by the vehicles. Number of research has been carried out earlier with respect to this system.

Over the years automatic car license plate recognition has gained lot of attention from the researchers. It has become one of the important systems for traffic monitoring and vehicles surveillance.

The car license plate recognition system is usually made up of following steps:

1. Capturing of license plate image: The moving car images or video is taken. In case of the video the

Video is preprocessed into still frames to provide car images.
2. Once the image is captured the next step is to detect the license plate from the whole image which may include car image too.

3. Once the boundary of the license plate is recognized the next step is to preprocess the image and segment the characters.

4. When each character is segmented the algorithm aims to recognize the segmented characters.

Over the years many systems have been successfully proposed and implemented. These systems made used of concepts of image processing, neural networks, classifiers, Support vector machines, Optical character recognition, etc. Each system possessed its own advantages and disadvantages. Literature review section explores them in detail. Based on the study, this thesis aims in enhancing the functionalities and accuracy of already systems in existence by integrating the concepts of SVM, Optical Character recognition and SURF.

The automatic license plate recognition system is to be developed for INDIAN car license system. Government of Delhi made an effort in launching an odd-even based car system wherein odd series cars are to be used on odd days and even series cars are to be used for even days. Thus, the proposed system is to be developed using the combination of optical character recognition using SURF and Multiclass State vector machine (SVM). This paper aims in developing a faster and accurate car license recognition system. IT further compares the proposed system with existing systems and other similar variants.

\section{LITERATURE REVIEW}

The main task in automatic car license plate detection system is the number plate detection and the character recognition. Various methods have been proposed with these respects. Following section examines the methods for number plate detection or recognition.

\subsection{Techniques for Automatic car number plate recognition system}

Various segmentation methods are discussed here:

"Ref. [1]" gave the concept of sliding concentric window method which is a two phase method where in the concentric windows are slides from upper left corner of the input image. Based on a set segmentation rule the statistical values for the both windows are computed. The rule states that if the ratios of the median of the given two windows are greater than the set threshold then the region of interest contains the center pixel. Once the whole images scanned the two windows stop scanning. This proposed system gave a success efficiency of 
$95 \%$ and the system took $112 \mathrm{~ms}$ for processing a number plate segmentation.

A similar kind of sliding concentric window based system is proposed and designed for Korean number plates by "Ref. [2]". The system made use of HIS based color model for the verification. Least square fitting method was used to rectify the tilt of the input image. This method was able to successfully segment the characters for the Korean number plate system.

"Ref. [3]" came up with a very fast algorithm for detecting the number plates. This algorithm was based on the frame detection module and was executed in three steps. In the first step the non-plate and plate regions are separated using the gradient feature methods. In the second step the complex plate regions are extracted and in the final step the plate verification is carried out such that no non- plate regions are extracted.

"Ref. [4]" came up with a feature based number plate localization method for the Indian license plate framework. The input images are converted to binary images using the Otsu's methods and based on the seven-step method the number plate is extracted from the total image of the vehicle.

In "Ref. [5]" salient features used to extract vehicle number plate. Salient features like color, shape and texture. Author used Hough transform (HT) to detect vertical and horizontal lines from rectangular vehicle number plate and then processed it by converting red, green, blue (RGB) to hueintensity-saturation(HIS). Finally, segmented number plate obtained.

Similarly for Indian system Ch.Jaya Lakshmi et al. "Ref. [6]" used the concept of texture based wavelets methods which helped to extract the characters of the license plate. "Ref. [7]" made usage of the image morphological operations and "Ref. [8]" used sobel masks which were used to detect the vertical edges. Such detection helped in enhancing better performances when considered in complex images with complex backgrounds. Similar sobel edge based detector operation was used by "Ref. [9]".

For detecting the license plate directly from a given CCTV footage "Ref. [10]" came up with a system which localized the given license plates captured from the video frameworks in sequence. The authors made use of a revised tracking and recognition techniques. The whole system works in four steps which includes computing connected components and finding contours, deciding the region of interest using rectangular based size, localization on the basis of the image histogram, mean classification and gradient based processing. Finally the tracking is done.

In "Ref. [11]" canny edge detector operator was applied to find out the transition points. As per H.ErdincKocer et al a license plate contains white background and black character normally. The Canny edge detector uses a filter, which is then based on Gaussian smoothing's first derivative to eliminate the noise. Then in the next step, the edge strength is calculated by considering the gradient of the image. The canny edge detector operator used $3 \times 3$ matrixes to accomplish this task. Based on this information transition points region is determined. The edge map is used to find transition points between black and white colors. The further technical details of this algorithm are not mentioned. The vehicle images were captures from CCD camera.

An enhanced segmentation method was given by "Ref. [12]" which is executed in four different steps which included median filtering, thresholding, labeling of the components, segmentation, noise filtering. The system proved to be a successful one.

\subsection{Discussion}

On examining the literature works and related works it can be said that the segmentation methods for the number plates are normally restricted to work in proper light conditions, shape of the number plate, color, distance from the camera, language, vehicle etc. thus only few real -time based algorithms are able to work on direct video rest all work on static images.

The main need is to have a good object tracking and detection. "Ref [13]" came up with an efficient SURF based object detection which has the highest efficiency for its objective.

Thus based on the analysis chose SURF based method for number plate recognition.

\section{METHODOLOGY}

This section explains the methodology used for car license plate detection system (CLPDS). "Figure 1"shows the basic block diagram of the whole system to be designed.

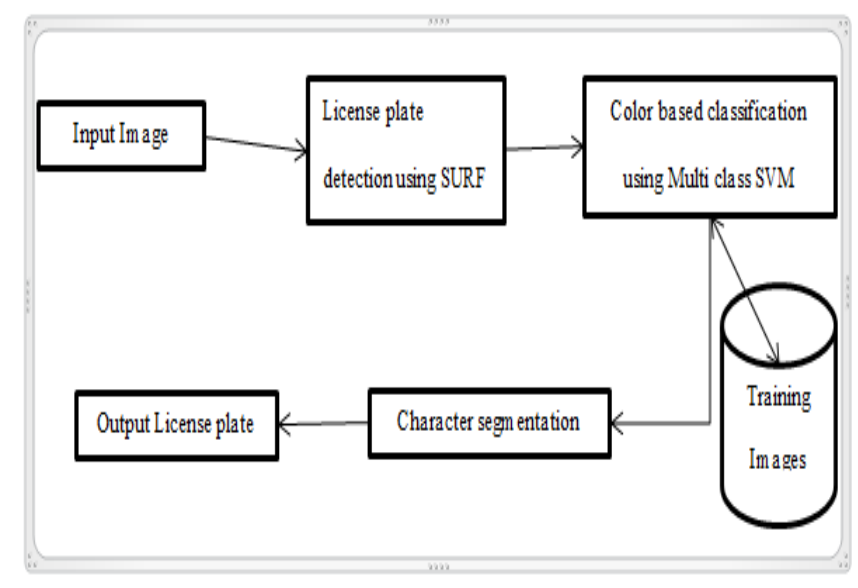

Fig 1: Block diagram of the system

\subsection{Algorithm 1: Main algorithm for CLPDS}

Step 1: Preprocessing module

Step 1.1: Create a database of images consisting of car and license plate.

Step 1.2: Create a database for color based classification of images.

Step1.2.1 divide this database in 70:30 ratio for training and testing respectively.

Step1.2.2 train the Multiclass SVM by setting histograms at normalized L1 norm using one vs all based classification.

Class labels for the Multi class SVM are as follows

Red color: "president vehicle"

Yellow color: "commercial vehicle"

White color: "private vehicle"

Step1.2.3: positive results indicate color based classification of the images and negative rest part of the 
image. Multiclass SVM is now trained for color based classification of license plate.

Step 2: enter the input image for license plate detection

Step 3: call SURF based image segmentation program. This identifies the license plate in the given image (Algorithm 2)

Step 4: call trained Multiclass SVM for color based classification. Display the result of classification in the form of class labels.

Step 5: For the detected license plate perform character segmentation and display the segmented image.

Step 6: Display the final read characters

\subsection{Algorithm 2: SURF based image segmentation}

This algorithm is used for segmenting the rest of the image with the car license plate. Thus, this algorithm displays the detected car license plate image. The detected image is displayed in rectangular box.

image1 (This is the image that is used as template to match)

Read the input image.

Convert the image to binary format by converting it into gray scale image.

Extract SURF interest points.

Obtain features.

Match features.

Get 25 strongest features.

Match the number of strongest features with each image.

Take the ratio of- number of features matched/ number of strongest features (which is 25).

If there are two images of the same object (two images taken separately on a camera), ideally the ratio should be near 1 or near $100 \%$.

In this algorithm one needs to give the images of car license plate as master image from the database and a manually cropped image of number as the test image. Now the algorithm begins by extracting the SURF points of the master image and stores them. Then the other part extracts the SURF points of the test image such that it also calculates similar points in both the images. After that each surf points is matched to the nearby similar SURF point. With the aid of the scale and orientation of the SURF point descriptor, each matched similar SURF point pair with its translation from the master image to the test image is displayed. Thus the object to be detected is bounded in a rectangular box and gets the detected characters and number detected from the whole image.

It is noted that 1 pair of correctly matched similar SURF points can help in deciding the position, scale and orientation of the object in the test image. Usually not all of the matched pairs are correct; therefore one has to use all of the pairs of projected surf points to cast votes on what are the correct position, scale and orientation of the car license number and even the usage of different font size in the test image. In this special scenario the outcome with the highest votes are then refined.

\section{EXRERIMENTATION \& RESULTS}

The methodology is implemented in MATLAB R2013 b environment. The described methodology is divided into the following step: Input the license plate image (see in figure2), Apply SURF process: this process displays the detected car license plate image. The detected image is displayed in rectangular box (see in figure 3). Then apply characters segmentation (see in figure 4). And then display the category as private vehicle, series as even series or odd series and the number of the license plate (see in figure 5).The experiment is conducted using a database of 120 license plate images. and obtained the accuracy of $94 \%$. The result of the experiment is shown in the "Table 1".

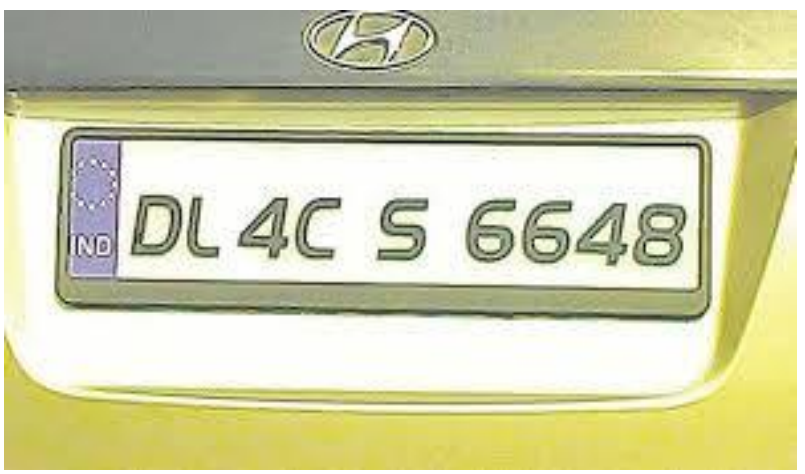

Fig 2: Input Image

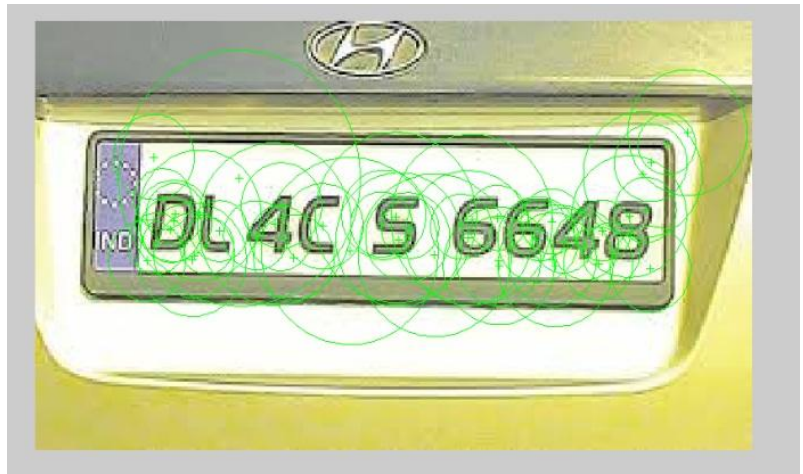

Fig 3: SURF Process

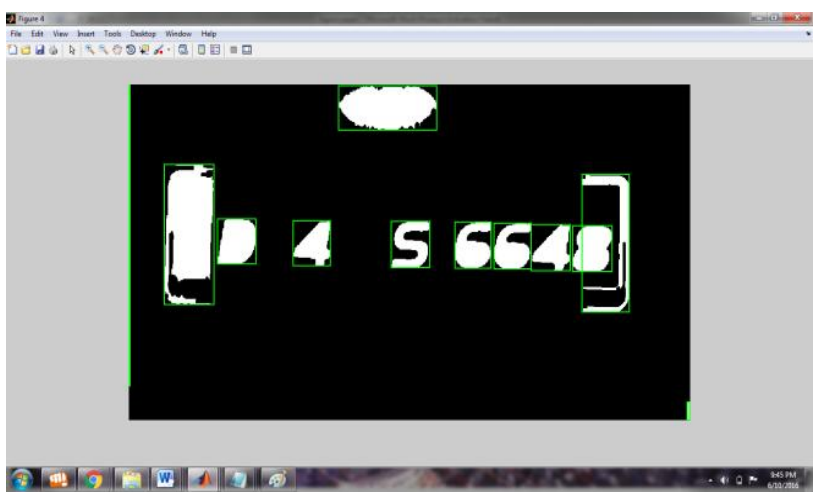

Fig 4: Character Segmentation Figure 


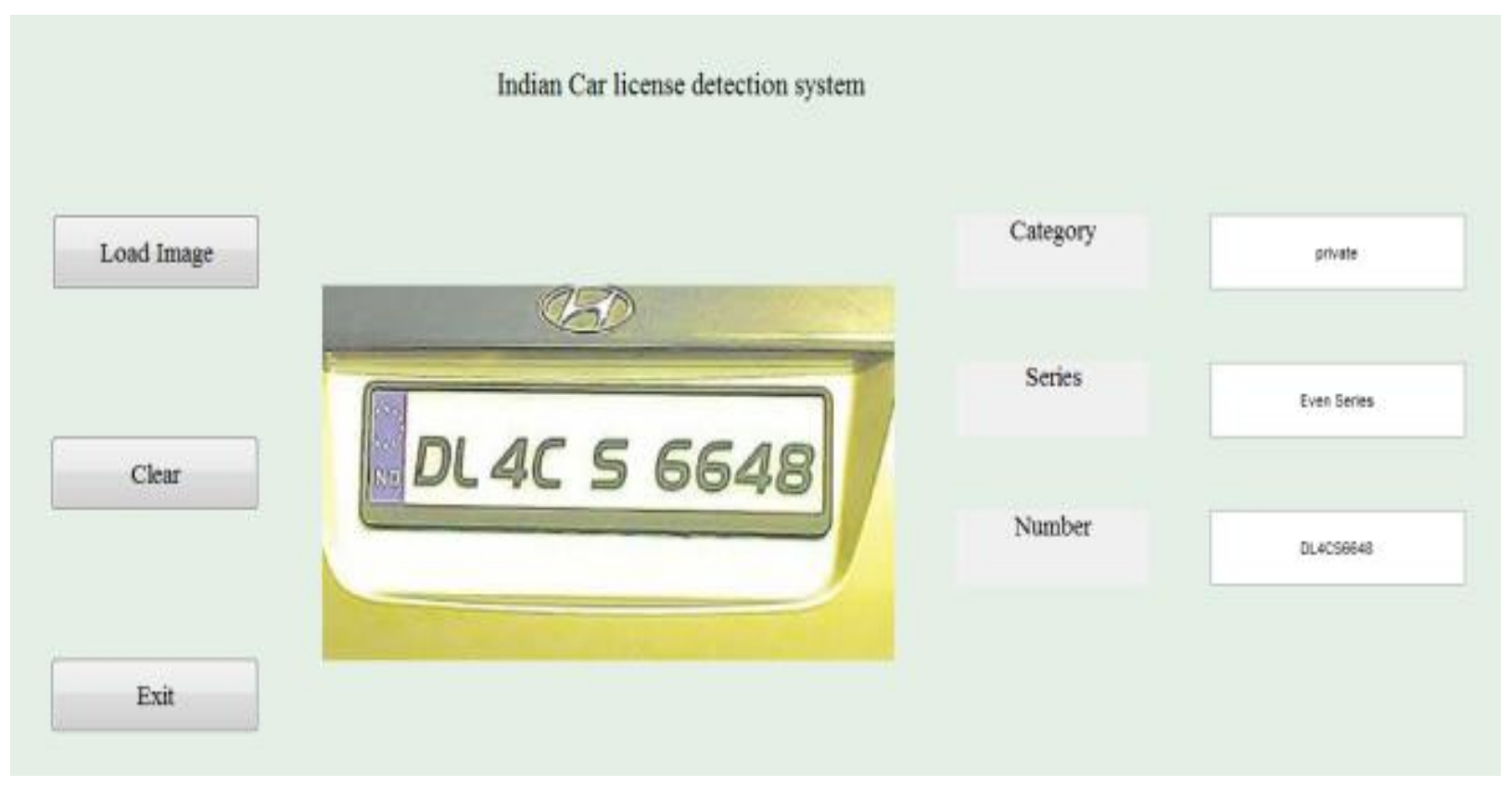

Fig 5: Final GUI Output

Table 1. Assessment of Result

\begin{tabular}{|c|c|c|c|c|c|c|}
\hline $\begin{array}{c}\text { License Plate } \\
\text { image }\end{array}$ & $\begin{array}{c}\text { Localization } \\
\text { status }\end{array}$ & $\begin{array}{c}\text { Character } \\
\text { Segmentation }\end{array}$ & $\begin{array}{c}\text { Character } \\
\text { recognition } \\
\text { results }\end{array}$ & $\begin{array}{c}\text { Error in } \\
\text { Alphabet }\end{array}$ & $\begin{array}{c}\text { Error in } \\
\text { Number }\end{array}$ & Odd/Even \\
\hline DL3BC4511 & SUCCESS & DL3BC4511 & DL3BC-4511 & NIL & NIL & ODD \\
\hline DL4CAF4943 & SUCCESS & DL4CAF4943 & DL4CAF-4943 & NIL & NIL & ODD \\
\hline DL4BA2322 & SUCCESS & DL4BA2322 & DL4BA-2322 & NIL & NIL & EVEN \\
\hline DL32A1234 & SUCCESS & DL32A1234 & DL32A-1234 & NIL & NIL & EVEN \\
\hline DL4AC3265 & SUCCESS & DL4AC3205 & DL4AC-3205 & NIL & 1 & ODD \\
\hline DL1CU9720 & SUCCESS & DLTCU9720 & DLTCU-9720 & 1 & NIL & ODD \\
\hline DL4CS6648 & SUCCESS & DL4CS6648 & DL4CS-6648 & NIL & NIL & EVEN \\
\hline DL9CW2806 & SUCCESS & D9W12806 & D9W1-2806 & 2 & NIL & EVEN \\
\hline HR26AW5000 & SUCCESS & HR26AV500 & HR26AV-500 & 1 & 1 & EVEN \\
\hline DL9CW2805 & SUCCESS & DL9CU2805 & DL9CU-2805 & 1 & NIL & ODD \\
\hline
\end{tabular}

\section{CONCLUSION}

Automatic license detection system is an essential system in today's busy traffic system. In India there are many cases of rash driving where in cars violating many traffic rules. It becomes very difficult for traffic policeman to capture the details of the vehicle. Thus, automatic license detection system have been proposed and implemented over the years to help easy and fast monitoring of traffic rules by the vehicles. Number of research has been carried out earlier with respect to this system.

The aim of this work is to build an automatic car license recognition system where in the car license data base is first classified into various categories of license Plates followed in India. This classification is done through multiclass SVM. Once the classification is done an integrated SURF and SVM based recognizer is used for character recognition of the license plate. The work also classifies the license plate as an odd-even series license plate based on the recognized numbers of the plate. The proposed method is built and implemented successfully.

It is found that for the selected database the system gave an accuracy of $94 \%$. The accuracy of the system was mainly reduced due to error in the recognition of the characters of the license plate. The efficiency of this work can be improved further by integrating an optical Character recognition system for multiple fonts so that varying styles of number plates can be recognized.

\section{REFERENCES}

[1] Christos Nikolaos E. Anagnostopoulos, Ioannis E. Anagnostopoulos, VassiliLoumos, and EleftheriosKayafas, "A License Plate-Recognition Algorithm for Intelligent Transportation System Applications," pp. 377-392, 2006.

[2] Kaushik Deb, Ibrahim Kahn, AnikSaha, and Kang-Hyun Jo, "An Efficeint Method of Vehicle License Plate Recognition Based on Sliding Concentric Windows and Artificial Neural Network," Procedia Technology, vol. 4, pp. 812-819, 2012.

[3] Shen-Zheng Wang and Hsi-Jian Lee, "A cascade framework for real-time statistical plate recognition system," IEEE Trans. Inf. Forensics security, vol. 2, no. 2, pp. 267-282, 2007. 
[4] PrathameshKulkarni, AshishKhatri, PrateekBanga, and Kushal Shah, "Automatic Number Plate Recognition (ANPR)," in RADIOELEKTRONIKA. 19th International Conference, 2009.

[5] Zhen-Xue Chen, Cheng-Yun Liu, Fa-Liang Chang, and Guo-You Wang, "Automatic License-Plate Location and Recognition Based on Feature Saliance," IEEE Transactions on Vehicular Technology, vol. 58, no. 7, pp. 3781-3785, 2009.

[6] Ch.Jaya Lakshmi, Dr.A.Jhansi Rani, Dr.K.Sri Ramakrishna, and M. KantiKiran, "A Novel Approach for Indian License Recognition System," International Journal of Advanced Engineering Sciences and Technologies, vol. 6, no. 1, pp. 10-14, 2011.

[7] AbdulkarSengur and YanhuiGuo, "Color texture image segmentation based on neutrosophic set and wavelet transformation ," Computer Vision and Image Understanding, vol. 115, no. 8, pp. 1134-1144, August 2011.

[8] Jiann-Jone Chen, Chun-Rong Su, W.E.L Grimson, JunLin Liu, and De-HuiShiue, "Object Segmentation of Database Images by Dual Multiscale Morphological Reconstructions and Retrieval Applications," IEEE Transactions on Image Processing, vol. 21, no. 2, pp. 828-843, 2012.
[9] MahmoodAshooriLalimi, SedighehGhofrani, and Des McLernon, "A vehicle license plate detection method using region and edge based methods," Computers \& Electrical Engineering, November 2012.

[10] M. S. Sarfraz et al., "Real-Time automatic license plate recognition for CCTV forensicc applications," Journal of Real-Time Image Processing- Springer Berlin/Heidelberg, 2011.

[11] H. ErdincKocer and K. KursatCevik, "Artificial neural netwokrs based vehicle license plate recognition," Procedia Computer Science, vol. 3, pp. 1033-1037, 2011.

[12] A Roy and D.P Ghoshal, "Number Plate Recognition for use in different countries using an improved segmenation," in 2nd National Conference on Emerging Trends and Applications in Computer Science(NCETACS), 2011, pp. 1-5.

[13] HuShuo, Wu Na, SongaHuajun, “Object Tracking Method Based on SURF", Elsevier, Conference on Modelling, Identification and Control. AASRI Procedia,Volume 3, 2012, pp. 351-356.

[14] PhalgunPandya, Mandeep Singh "Morphology based approach to recognize Number plate in India" International Journal of Soft Computing and Engineering (IJSCE)ISSN: 2231-2307, Volume-1, Issue-3, July 2011. 